\title{
A TRAIL of destruction
}

Tumor cells that become chemoresistant are a major cause of relapse in patients with cancer. Specifically attacking chemoresistant cancer cells could combat resistance and improve responses to cancer treatment.

Following the observation that cancers contain stem-like cells that can reinitiate tumorigenesis with increased resistance to conventional treatment, Loebinger and colleagues investigated a therapy that selectively targets these cells. "We are studying a novel form of therapy that uses mesenchymal stem cells to carry the anticancer cell molecule TRAIL to tumors," describes co-investigator Sam Janes.

Owing to their ability to target tumors throughout the body, mesenchymal stem cells can be used to deliver anticancer therapy directly to cells in the tumor. Janes and coworkers engineered mesenchymal stem cells to express TRAIL (TNF-related apoptosis-inducing ligand), a protein that triggers apoptosis in cancer cells but not in normal cells.
The investigators assessed the effect of this anticancer therapy in cell cultures. As expected, the TRAIL-expressing mesenchymal stem cells specifically targeted tumors, killed cancer cells, and reduced the risk of relapse.

Janes notes that "this therapy has equal efficacy on both parent populations of cancer cells and the chemoresistant stemlike population." These cells underwent apoptosis, death, and reduced colony formation following exposure to the TRAIL-expressing mesenchymal stem cells. Janes adds that "this novel therapy attacks chemoresistant cancer cells possibly flagging this therapy as an adjunct to traditional therapeutic regimens that are currently hindered by chemoresistance." The researchers plan to continue developing and improving this technique.

\section{Lisa Richards}

Original article Loebinger, M. R. et al. TRAIL-expressing mesenchymal stem cells kill the putative cancer stem cell population. Br. J. Cancer 103, 1692-1697 (2010) 